
D:\Nsurg \Vol. 24, No. 2, Apr. - Jun., 2020\Nsurg-7.Doc
Fig. 1 Color
(A)
P. $187-194$
II

ORIGINAL ARTICLE

\title{
Assessment of Nursing Care Skills in Neonatal Unit: A Cross Sectional Observational Study
}

\author{
RUKHSANA RAFIQUE, MUHAMMAD HUSSAIN, MUHAMMAD AFZAL \\ SYED AMIR GILANI \\ The School of Nursing, The University of Lahore - Pakistan
}

DOI 10.36552/pjns.v24i2.443

\begin{abstract}
Objective: Increased hospital treatment is required to reduce neonatal mortality in low/middle-income (LMIC) countries. Nurses are vital for providing safe and efficient treatment, but a shortage of nurses and high patient workloads can lead to missed treatment. We aimed to evaluate thecare provided to newborns and finding missing care using direct observation methods.
\end{abstract}

Material and Methods: Cross sectional observational study. The sample size was 133 which were calculated through Slovin's formula. An adopted research tool was used which contain two sections. Section I included demographic data of nurses and neonates admitted in the ward. Section IIwas an Observational checklist on neonate's care with "Yes" "NO" which include 5 main themes that had sub points.

Result: Handing over the baby was less fifty five percent. Member not assesses the patient at the end of shift $54.1 \%$ nurses done this and $45.1 \%$ missed this task. Intravenous drug administration with a septic technique was 42 percent. Counselling of kangaroo mother care was 45.9 percent done and 54 percent missed.

Conclusion: Research addresses a significant gap in global literature regarding quantification of nurse's treatment using direct observational methods. We found considerable variability in the implementation of the task with potentially significant consequences for well-being and health of patients. The concentration of nursing tasks in babies was less than $60 \%$ on average. Tertiary care setting environment lacks a large proportion of nursing care with potentially serious effects on patient health and outcomes.

Keywords: Nurses, Practices, Neonate Care.

\section{INTRODUCTION}

Nursing is the care that is individualized for each neonate. ${ }^{11}$ Early neonatal death (ENND), described as the death of a newborn between 0 and 7 days after birth, accounts for globally $73 \%$ of all postnatal deaths. In high-income nations, however, prematureness and congenital defects are the main causes. ${ }^{10} \mathrm{In}$ Pakistan neonatal mortality rate $(\mathrm{NMR})^{\wedge}$ is 46 deaths per 1,000 live births. ${ }^{5}$

The highest impact on neonatal deaths will come from work-related and birth-related activities such as emergency obstetric treatment and pre-labor management, and care for young and old, such as resuscitation and neonatal infection control. ${ }^{13}$
Enhanced hospital care is needed in low-/middleincome (LMIC) countries to reduce newborn mortality. More than 60 percent of all newborns have neonatal jaundice. Phototherapy has an impact in neonatal intensive care unit treating all cases of jaundice. Therefore, during exposure to phototherapy, the nurse will cover neonate eyes to avoid retinal damage, give extra fluid, monitor vital signs and detect possible side effects and assess progress. ${ }^{8}$

Oxygen is the most widely used drug at the NICU and is considered to be the main component of nursing success, it is important to study the performance of nurses with regard to oxygen therapy and to conduct in-service training courses to avoid potential long-term 
complications. $^{7}$

The level of vital signs monitoring for children admitted to tertiary hospitals changing in their geographical setting and mortality rate.Precise intake measurements are sometimes lacking, especially for respiratory and pulse rate, likely linked to manual calculation. The level of monitoring is often small in the high-risk people. Studies possibly suggesting how the standard of care is compromised via significant shortages of human resources. ${ }^{15}$

Evaluations of services in low-resource settings have shown that in many situations systems are in place to support the provision of neonatal emergency care, but staff are unable to provide all of emergency care signaling functions. Lack of expertise, along with shortages of health workers, is likely to be a key reason why many critical services in low-resource environments are not provided or sub-optimally administered to mothers and neonates. ${ }^{18}$ While quality care delivery being based on health worker skill, that are rarely evaluated. Where assessments were carried out, most of these were urgent treatment of newborns in public-sector hospitals, with less recorded care of young and sick babies and private facilities. ${ }^{13}$

Although there are many literatures on missed care, but maximum of these were from the high income setting and literature on missed care mostly focus on one or two care delivered by nurses. In present study main focus is on observational method, secondly it also involves all the aspect of neonate.

\section{MATERIAL AND METHODS}

\section{Study Design and Sample Size}

It was a cross sectional observational study. Research was completed between December, 2019 to March, 2020. This study was conducted in Lahore General Hospital Lahore - a tertiary care hospital.The 200 staff nurses working in the neonate's unit providing care to the neonates admitted in ward, ICU, Nursery and emergency. Sample size calculated by Slovin's formula; $\mathrm{N} /\left(1+\mathrm{N}(\mathrm{e})^{2}\right)$. The sample size was 133.A convenient sampling technique was used to collect data.

\section{Inclusion Criteria}

The nurses present at the time of data collection and providing direct care to the neonates, nurses aged between $21-60$ years, and nurses that have at least 6 - month experience in neonates' unit.

\section{Exclusion Criteria}

The nursing administrator, nursing assistant, Doctors and paramedical staff. An adopted research tool was used which contain two sections. Section I included demographic data of nurses and neonates admitted in the ward. Section II was an Observational checklist on neonate's care with "Yes" "NO" which include 5 main themes that had sub points. ${ }^{6}$ A pilot study was carried out on 10 percent of nurses in order to test the clarity of the checklist and to estimate the time needed to fill the sheet. Crohn Bach Alpha was 0.85. By the use of tool II each nurse observed three times, once every shift. The time required to observe each nurse varied between $30-45$ minutes. Practice score of the total nurses was developed. Each item observed has been verified as satisfactory or unsatisfactory. Total score 100 and each item contain 2 points. Each item given and distributed as follow; Routine task (26point), Regular task (16 point), Critical task (34 points), Intravenous fluid Administration (8 point), Kangaroo mother care (4 point), Documentation of task (12 points). Total practice score of nurses was categorized as follows: Unsatisfactory < 60\%, satisfactory $>60 \% .{ }^{11}$

\section{Data Analysis}

The Social Sciences Statistical Package (IBM SPSS Statistics) versions 25 was used to calculate the results. To learn the distribution of data in demographic data and each statement, descriptive analysis was first computed. Then checklist statements were computed.

\section{RESULTS}

\section{Age Incidence}

Table 1 show that most of people fall in the age category $21-30$ years with $40.6 \%$ and minimum participants in the age of $51-60$ years with $5.3 \%$. No

Table 1: Demographic Data

\begin{tabular}{|c|c|c|}
\hline & Frequency & Percentage \\
\hline \multicolumn{3}{|l|}{ Age } \\
\hline $21-30$ & 54 & $40.6 \%$ \\
\hline $31-40$ & 53 & $39.8 \%$ \\
\hline $41-50$ & 19 & $14.3 \%$ \\
\hline $51-60$ & 7 & $5.3 \%$ \\
\hline
\end{tabular}


Rukhsana Rafique, et al

\begin{tabular}{|l|c|c|}
\hline \multicolumn{3}{|l|}{ Education } \\
\hline $4-$ years diploma & 72 & $54.1 \%$ \\
\hline BSN (Post RN) & 57 & $42.9 \%$ \\
\hline BSN Generic & 4 & $3.0 \%$ \\
\hline Master in Nursing & 0 & $0 \%$ \\
\hline Working Experience & & \\
\hline $1-10$ years & 84 & $63.2 \%$ \\
\hline $11-20$ years & 49 & $36.8 \%$ \\
\hline $21-30$ years & Nil & $0 \%$ \\
\hline More than 30 years & 0 & $34.6 \%$ \\
\hline Child Category & 46 & $0 \%$ \\
\hline Critical/HDU) &
\end{tabular}

\begin{tabular}{|l|l|l|}
\hline Emergency/Acute & 54 & $40.6 \%$ \\
Wards & 33 & $23.8 \%$ \\
\hline
\end{tabular}

one had an MSN degree qualification.3\% participants have BSC generic and $54.1 \%$ were 4 - year's diploma nurses. Maximum nurses were experienced in $1-10$ years $(63.2 \%) .36 .8 \%$ nurses have $11-2$ years' experience. No one had experience more than 20 years. A patient that was observed has different percentages $34.6 \%$ were in ICU and HDU, $40.6 \%$ in emergency and $24.8 \%$ were admitted in wards.

Table 2 shows different result, some task has a very good practice and some have very bad practice about the task. In routine task, handing over the neonates shows that $55.6 \%$ done this task and $44.4 \%$

Table 2: Observational Checklist.

\begin{tabular}{|c|c|c|c|}
\hline $\mathbf{S} / \mathbf{N}$ & Task & Done & Not Done \\
\hline \multicolumn{4}{|c|}{ Routine Task } \\
\hline 1. & Handing over baby between shift & $74(55.6 \%)$ & $59(44.4 \%)$ \\
\hline 2. & Patient assessments performed at the end of each shift. & $72(54.1 \%)$ & $61(45.1 \%)$ \\
\hline 3. & Cleaning of baby as required. & $65(48.9 \%)$ & $68(51.1 \%)$ \\
\hline 4. & Change linen of baby. & $69(51.9 \%)$ & $64(48.1 \%)$ \\
\hline 5. & Checking incubator settings daily. & $67(50.1 \%)$ & $66(49.6 \%)$ \\
\hline 6. & Hand washing/ Hand rub using sanitizer after touching the neonates. & $65(48.9 \%)$ & $68(51.1 \%)$ \\
\hline 7. & Checking cannula site at the start of shift & $60(45.1 \%)$ & $62(54.1 \%)$ \\
\hline 8. & Providing Cord care when required. & $57(61.2 \%)$ & $75(56.4 \%)$ \\
\hline \multicolumn{4}{|c|}{ Regular Tasks } \\
\hline 9. & Checking vital sign regularly. & $69(51 . \%)$ & $64(48.1 \%)$ \\
\hline 10. & Temperature checking every 2 hours. & $71(53.4 \%)$ & $62(46.6 \%)$ \\
\hline 11. & Check pulse for 1 mint. & $63(47.4 \%)$ & $70(52.6 \%)$ \\
\hline 12. & Check Respiratory during checking vital sign. & $70(52.6 \%)$ & $63(47.4 \%)$ \\
\hline \multicolumn{4}{|c|}{ Critical Tasks } \\
\hline \multicolumn{4}{|c|}{ Nasogastric tube feeding } \\
\hline 13. & Checking for correct position of feeding tube. & $60(45.1 \%)$ & $73(54.9 \%)$ \\
\hline 14. & Checking gastric aspirate before feeding. & $71(53.4 \%)$ & $62(46.6 \%)$ \\
\hline 15. & Checking actual volume of feeds. & $68(51.1 \%)$ & $65(48.9 \%)$ \\
\hline 16. & Positioning baby after feeding. & $64(48.1 \%)$ & $69(51.9 \%)$ \\
\hline
\end{tabular}




\begin{tabular}{|c|c|c|c|}
\hline \multicolumn{4}{|c|}{ Phototherapy } \\
\hline 17. & Turning/positioning the baby every 2 hours. & $68(51.1 \%)$ & $65(48.9 \%)$ \\
\hline 18. & Skin assessment at least 2 times per shift. & $70(52.6 \%)$ & $63(47.4 \%)$ \\
\hline 19. & Check eye for damage from phototherapy. & $61(45.9 \%)$ & $72(54.1 \%)$ \\
\hline 20. & Changing eye pad once in duty shift. & $59(44.4 \%)$ & $74(55.6 \%)$ \\
\hline \multicolumn{4}{|c|}{ Oxygen therapy } \\
\hline 21. & Checking nostril tube position and nostril-care, at the start and end of shift. & $65(48.9 \%)$ & $68(51.1 \%)$ \\
\hline 22. & Checking Regulating oxygen flow. & $64(48.1 \%)$ & $69(51.9 \%)$ \\
\hline \multicolumn{4}{|c|}{ Intravenous drug administration } \\
\hline 23. & Dilutions and checking compatibility of drug. & $73(54.9 \%)$ & $60(45.1 \%)$ \\
\hline 24. & Review of treatment sheet before drug administration. & $65(54.9 \%)$ & $68(45.1 \%)$ \\
\hline 25. & Flushing cannula before administering drug. & $66(49.1 \%)$ & $67(50.4 \%)$ \\
\hline 26. & Administrating medication with a septic technique. & $56(42.1 \%)$ & $77(51.9 \%)$ \\
\hline 27. & Flushing cannula after giving medication. & $64(48.1 \%)$ & $68(51.5 \%)$ \\
\hline \multicolumn{4}{|c|}{ Intravenous Fluid administration } \\
\hline 28. & Review of treatment sheet before fluid administration. & $68(51.1 \%)$ & $65(48.9 \%)$ \\
\hline 29. & Intravenous fluid regulates as required. & $63(47.4 \%)$ & $70(52.6 \%)$ \\
\hline 30. & Flushing cannula before starting the fluids. & $70(52.6 \%)$ & $63(47.4 \%)$ \\
\hline \multicolumn{4}{|c|}{ Kangaroo Mother Care } \\
\hline 31. & Counseling and Support mother to initiate and continue with kangaroo mother care. & $61(45.9 \%)$ & $72(54.1 \%)$ \\
\hline 32. & Supervision of mother for correct Kangaroo mother care practice. & $64(48.1 \%)$ & $69(51.9 \%)$ \\
\hline
\end{tabular}

nurses not did this task. Observational study shows that every member not assess the patient at the end of shift $54.1 \%$ nurses done this and $45.1 \%$ missed this task. Cannula site checking is a very important nursing task that was not performed by every client $45.1 \%$ clients done this and $54.1 \%$ nurses missed this task. Neonates cord care is an essential nursing task, but not every nurse does this $61.2 \%$ applicants do this and $56.4 \%$ applicants missed this task. Some tasks are regular task that was not performed by everyone. Regular task includes vital sign, temperature, respiration, checking pulse, etc. Result of the present study was $51.9 \%$ checked vitals and $48.1 \%$ missed this task. 53.4\% check temperature and $46.6 \%$ did not check temperature. Respiration rate checking missed by $47.4 \%$ applicants and $52.6 \%$ applicants did this task. Critical task has different subpoints and have different percentage regarding this task. In nasogastric tube feeding $53.4 \%$ participants check aspiration before feed and $46.6 \%$ participants missed this task. $48.1 \%$ not position the baby after feeding through a

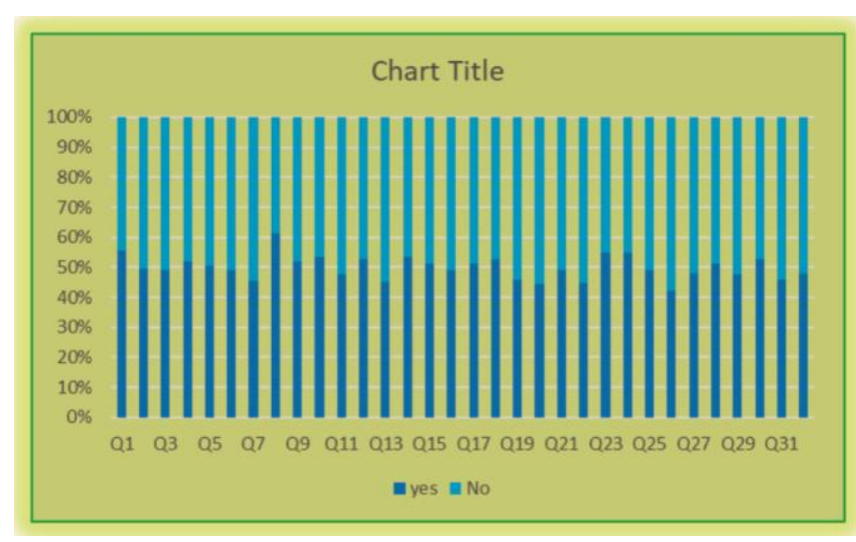

Graph Chart of Nurses Practices. 
nasogastric tube. $54.4 \%$ members did not check the correct position of neonate. $51.1 \%$ members turned the baby every 2hours during Phototherapy 52.6\% checked the skin phototherapy. $54.1 \%$ clients checked the eye during phototherapy and $55.6 \%$ clients did not change the eye pad during phototherapy section. $48.9 \%$ participants checked nostril position and nostril care. 48.1\% participants checked oxygen flow regulating and $51.9 \%$ did not perform this task.54.9\% clients checked the drug compatibility and $45.1 \%$ did not check it. $54.9 \%$ participants reviewed the treatment sheet when they administer drug and $45.1 \%$ missed this practice. Participants had a very wrong practice about aseptic technique when they administer drugs. $51.5 \%$ participants not flush the cannula and $48.1 \%$ not flush the cannula after medicine. 52.6\% participants regulate fluid when they required $47.4 \%$ not regulate it. $51.1 \%$ participants review treatment chart when they administer fluid and $48.9 \%$ missed this task. $54.1 \%$ participants did not support the kangaroo mother and $45.9 \%$ support the mother. $51.9 \%$ participants did not supervise the kangaroo mother care and $48.1 \%$ supported this practice.

\section{DISCUSSION}

The purpose of this study was to quantify nursing tasks that could be observed for newborns and identify missed care. Several practices were observed and identify that was not delivered to the neonates. Overall score for practices was unsatisfactory (49.3\%). Nurses working in high black neonatal ICU missed nearly fifty percent nursing care due to high patient to nurse ratio. Another study also had similar results that nurses have an unsatisfactory practice in the neonate unit. ${ }^{9}$ Hand hygiene practices are very important nursing task to prevent from infection among neonates and premature. Present study showed that (48.9\%) nurses wash hands before and after touching the patient. ${ }^{2}$ Showed that the respondents' overall attitude towards hand hygiene was unsatisfactory, showing a weak moderate attitude $(54.73 \%)$. More than ninety percent of healthcare workers were aware of hand hygiene in avoiding healthcare-associated infection and ninetyone point one percent of them could improve their compliance with hand hygiene. There is another study shows that $75 \%$ nurses' compliance with hand hygiene this result disagree with both these studies. In recent study hand over patient between shift is a task that was not completely done (44.4\%) of this task left by nurses. Based on an interservice exploratory review, interprofessional transfers between Obstetric nursing and NICU teams, we found that fifty two percent of the transfers skipped one or more elements of clinical content. ${ }^{4}$ In our study showed that drug diluting the more than one drug and checking the compatibility of drug is $54.9 \%$ that was high. A study conducted at the University of Sydney in 2010 regarding the interruption of medication result of the study was similar 53.1\% .of all administrations. ${ }^{20}$

Cleaning the baby and changing the linen of the baby is another nursing task that was not completely done in recent study another observational study shows that nurses only clean the baby that was sick and left those that was stable and not sick for their mother to clean the baby. ${ }^{14}$ In recent study sixty one percent nurses wash cord according to another study seventy percent nurses wash cord there is a little difference in both results. ${ }^{4}$

Present study showed that Flushing cannula before and after drug administration was 48.1 and 49.1 percent respectively. There was another study showed that practices of that study participants was 34 percent that is lower than present study. ${ }^{19}$ Present study showed that $53 \%$ nurses checked the temperature regularly according to study criteria, but this practices was unsatisfactory. A another study showed opposite result where, nurses showed good practice $98.6 \%$ relate to checking temperature. ${ }^{6}$

Checking vital sign is a key task of nursing practice present study showed that complete vital sign checking ratio is range up to fifty percent another observational study conducted in Kenya in 2018 showed that record of full set of vital signs is fifty seven percent and that is near to present study. ${ }^{15}$ Another study showed that $70 \%$ nurses check standard rules and half of them wash incubator inside and outside every 8 hour to prevent from infection. ${ }^{4}$

Current study showed the nursing practice regarding the correct nasogastric tube position, (45.1\%), gastric aspiration before feed $53.4 \%$, actual volume of feed $51.1 \%$. An observational studyon ${ }^{17}$ nursing practice on nasogastric tube placement and feeding showed that correct position includes fifty percent, eighty three percent used fluid aspiration this result was more than current study. A study ${ }^{12}$ showed that more than half of nurses has a high level perception regarding enteral feeding. Some studies reported that nurses had an unsatisfactory level of practice related to nasogastric tube feeding, checking the fluid aspiration, correct position checking the actual volume of fluid before administration. ${ }^{14,16}$ 
Present study agrees with the result of this because present study also has unsatisfactory level of practice related to nasogastric feeding.

Present study showed that nurses had unsatisfactory practice regarding phototherapy. Another study ${ }^{1}$ had the opposite result showed that nurses have satisfactory knowledge and practice regarding phototherapy. Another study also showed the opposite result that maximum nurses had satisfactory practice related to care of the neonate who receive phototherapy care. Two-point five percent nurses had an unsatisfactory level of care related to diaper eye and bottle feeding this result agree with the present study. ${ }^{23}$

Current study showed that nurses working in Kangaroo mother department have unsatisfactory practice related to kangaroo mother care. Another study showed similar practices. ${ }^{3}$ This study showed that nurses had adopted a poor practice related to kangaroo mother care.

In the present study, the nurses had an unsatisfactory practice regarding the oxygen flow. Another study showed that nurses did know about the long-term oxygen administration. Their study showed that there is a lack of guideline for oxygen administration. ${ }^{16}$ Another study showed that nurses had favorable performance $39.1 \%$ regarding oxygen therapy. ${ }^{7}$ The results of another study somehow agreed with this study according to them $42 \%$ of nurses has inadequate practice related to administration of oxygen therapy.

\section{CONCLUSION}

Our research addressed a significant gap in global literature regarding the quantification of nurse's treatment using direct observational methods. We found considerable variability in the implementation of the task with potentially significant consequences for well-being and health of patients. The concentration of nursing tasks in babies was less than $60 \%$ on average. Improving the standard of care and its contribution to neonatal survival obviously needs an expansion of the nursing workforce, potentially complemented by additional advances in human resources. Failure to tackle key problems in the workforce would result in missed treatment remaining widespread and weaken attempts to provide highimpact, low-cost services for small and sick children.

\section{Recommendations}

Based upon the result of the current study, the following recommendations can be construed. The ratio of nurses and neonate should be 1:2. Pre-service educational and training for newly employed nurses will help to update and advance their practice. In service training curricula lead toward all characteristics of care provided for neonates should be directed. Head nurses 'should supervise the practices of nurses and proper feedback should be given. Booklets for life-threatening procedures for premature neonates should be present in the unit. ${ }^{11}$

\section{Ethical Consideration}

The approval for ethics was received from university of the Lahore Ethics Review Committee and permission letter was received from the concerned authority of tertiary hospital to conduct study in this setting. Hardcopies of the checklist will be held in a locked cabinet that was only open to approved personnel, and softcopies were encrypted while keeping on the desktop of the Principal Investigator.

\section{Limitation}

Due to the single setting result cannot be generalized. Despite our effort we cannot find out the rules why nurses' change polices of care during observational study. The study has short time period and a small sample size.

\section{REFERENCES}

1. Ahmed SM, Hani M. Assessment of Nurse's Knowledge and Practice Working in District Hospitals at Minia Governorate about Neonatal Hyperbilirubinemia. IOSR. J. Nurs. Health Sci. 2017; 6 (2): 9-16.

2. Abduawahid MK, Mahmood NR. Awareness, Knowledge and Attitude of Hand Hygiene Practices among Healthcare workers in Kirkuk Pediatric Hospital. Middle East Journal of Family Medicine. 2020; 18 (2).

3. Almazan JU, Cruz JP, Albougami AS, Alamri MS, Adolfo CS. Maternity-ward nurses' kangaroo mother care attitudes and practices: implications and future challenges. Scandinavian journal of caring sciences, 2019; 33 (4): 848-56.

4. Ali LM, Ahmed DM, Mohamed SO, Mohammed MA (2019). Nurse's Skills Regarding Care of Preterm Infants in Neonatal Intensive Care Unit Selected in Jazan, KSA. Neonat Pediatr. 2019; 5: 184. 
5. Arora A, Kannampallil T, Abraham J. Interdisciplinary handover between obstetric nursing and neonatal physician teams: an observational study. BMJ Paediatrics Open, 2019; 3 (1).

6. Chandra Shekar M, Williams S. A Study to Assess the Knowledge and practice of staff Nurses Regarding Thermoregulation of Neonates selected Hospital at Mysuru. Asian Journal of Nursing Education and Research, 2018; 8 (1): 94-8.

7. Data from UNICEF, maternal and newborn health Disparities in Pakistan, 2015.

8. Gathara D, Serem G, Murphy GA, Abuya N, Kuria R, Tallam E, English M. Quantifying nursing care delivered in Kenyan newborn units: protocol for a cross-sectional direct observational study. BMJ Open, 2018; 8 (7): e022020.

9. HematiZ, Mohammadi R, Boroumand S, Poorpooneh Z, Ghazavi Z. Nurse' Performance Oxygen Therapy for Infants Hospitalized at the Neonate Intensive Care Unit.

10. Hockenberry MJ, Wilson D. Wong's Essentials of Pediatric Nursing9: Wong's Essentials of Pediatric Nursing. Elsevier Health Sciences; 2013.

11. Lake ET, Staiger D, Edwards EM, Smith JG, Rogowski JA. Nursing care disparities in neonata intensive care units. Health services research, 2018 Aug; 53: 3007-26.

12. Lehtonen L, Gimeno A, Parra-Llorca A, Vento M. Early neonatal death: a challenge worldwide. InSeminars in Fetal and Neonatal Medicine, 2017; 22, (3): 153-160. WB Saunders.

13. Mohamed BM, El Dakhakhny AM, Bassam SE, El Sayed LM. Assessment of Nursing Care Provided to Premature Neonates at Neonatal Intensive Care Unit at Zagazig University Children Hospital. Zagazig Nursing Journal, 2019; 7 (2): 1-4.

14. Mohammed AQ. Assessment of Nurses' Knowledge and Practices Regarding Nasogastric Tube at Neonatal Intensive Care Unit in Baghdad Hospitals. Indian Journal of Public Health Research \& Development, 2019; 10 (4): 585-91.

15. Mayhob MM. Nurses' Knowledge, Practices and Barriers Affecting a Safe Administration of Oxygen Therapy.

16. Metwaly EA, Mohammed EH, Mohammed MA. Nurses' Performance Regarding Nasogastric Tube Feeding in Intensive Care Units. Zagazig Nursing Journal, 2013; 9 (1): 69-86.

17. Murphy GA, Gathara D, Mwaniki A, Nabea G, Mwachiro J, and Abuya N, English M. Nursing knowledge of essential maternal and newborn care in a high mortality urban African setting: A cross sectional study. Journal of Clinical Nursing, 2019; 28 (5-6): 88293.

18. Nzinga $\mathrm{J}$, McKnight $\mathrm{J}$, Jepkosgei $\mathrm{J}$, English $\mathrm{M}$. Exploring the space for task shifting to suppor nursing on neonatal wards in Kenyan public hospitals. Human Resources for Health, 2019; 17 (1): 18.

19. Ogero M, Ayieko P, Boniface Makone TJ, Malla L, Oliwa J, Irimu G, English M. An observational study of monitoring of vital signs in children admitted to Kenyan hospitals: an insight into the quality of nursing care? Journal of global health. 2018; 8 (1).

20. Omer IM, Ibrahim NG, Nasr AM. Oxygen therapy in neonatal intensive care units in Khartoum State. Sudanese journal of Paediatrics, 2015; 15 (2): 49.

21. Parker LA, Withers JH, Talaga E. Comparison of neonatal nursing practices for determining feeding tube insertion length and verifying gastric placement with current best evidence. Advances in Neonatal Care. 2018; 18 (4): 307-17.

22. Regeru RN, Chikaphupha K, Bruce Kumar M, Otiso L, Taegtmeyer M. 'Do you trust those data?'-a mixedmethods study assessing the quality of data reported by community health workers in Kenya and Malawi. Health Policy and Planning, 2020.

23. Ramdan AA, Refat NH, Mobarak AA. Assessment of Nursing Practice Regarding Neonates with Hyperbilirubinemia. Assiut Scientific Nursing Journal, 2019; 7 (19): 52-60.

24. Randle J, Firth J, Vaughan N. An observational study of hand hygiene compliance in paediatric wards. Journal of Clinical Nursing, 2013; 22 (17-18): 2586-92.

25. Samantha Keogh RN, Caroline Shelverton RN, Julie Flynn RN, Karen Davies RN, Nicole Marsh RN, Rickard CM, Keogh S. An observational study of nurses' intravenous flush and medication practice in the clinical setting.

26. Westbrook JI, Woods A, Rob MI, Dunsmuir WT, Day RO. Association of interruptions with an increased risk and severity of medication administration errors. Archives of Internal Medicine, 2010; 26; 170 (8): 68390.

27. Zaki A, El-Sayed E, Said K, Ali R. Assessment of Nurses' Performance Regarding Care for Neonates with Necrotizing Enterocolitis at Intensive Care Units. Egyptian Journal of Health Care, 2018; 9 (3): 111-24. 


\section{Additional Information}

Disclosures: Authors report no conflict of interest.

Ethical Review Board Approval: The study was conformed to the ethical review board requirements.

Human Subjects: Consent was obtained by all patients/participants in this study.

Conflicts of Interest:

In compliance with the ICMJE uniform disclosure form, all authors declare the following:

Financial Relationships: All authors have declared that they have no financial relationships at present or within the previous three years with any organizations that might have an interest in the submitted work.

Other Relationships: All authors have declared that there are no other relationships or activities that could appear to have influenced the submitted work.

Address for Correspondence:

Rukhsana Rafique

Email: rukhsanarafique07@gmail.com

\section{AUTHORSHIP AND CONTRIBUTION DECLARATION}

\begin{tabular}{|c|c|c|}
\hline Sr.\# & Author's Full Name & Intellectual/Contribution to Paper in Terms of: \\
\hline 1. & Rukhsana Rafique & 1. Main investigator/ data collection. \\
\hline 2. & Muhammad Hussain & 2. Literature review \\
\hline 3. & Muhammad Afzal & 3. Co investigator and data collector. \\
\hline 4. & Syed Amir Gilani & 4. Analysis of data and quality insurance \\
\hline
\end{tabular}

Date of Submission: 8-4-2020

Date of Revision: 30-04-2020

Date of Online Publishing: 30-06-2020

Date of Print: 30-07-2020 